\title{
The antifibrotic drug pirfenidone inhibits spondyloarthritis fibroblast-like synoviocytes and osteoblasts in vitro
}

\author{
Julie Stougaard', Søren Lomholt ${ }^{1}$, Pernille Ommen², Jens Kelsen ${ }^{3}$ and Tue W. Kragstrup ${ }^{1,4,5^{*}}$ (])
}

\begin{abstract}
Background: The pathogenesis of spondyloarthritis (SpA) involves both inflammation and new bone formation in the spine. In line with this, the disease has been characterized as both inflammatory and fibrotic. The current treatment dampens inflammation while new bone formation can progress. Therefore, there is an unmet therapeutic need for the treatment of new bone formation in SpA. Fibrosis is mediated by myofibroblasts and new bone formation is the result of increased osteoblast mineralization and decreased osteoclast resorption. Here, we evaluate the potential effect of the newly approved anti-fibrotic agent pirfenidone (PFD) on fibrosis and new bone formation in cell culture models of SpA.
\end{abstract}

Methods: Fibroblast-like synoviocytes (FLSs) were isolated from SpA patients $(n=6)$ while the osteoblast cell line Saos-2 was purchased. The cells were cultured with PFD at 0.250 .5 , or $1.0 \mathrm{mg} / \mathrm{ml}$. The proliferation of FLSs was analyzed with light microscopy and flow cytometry. The differentiation and activation of FLSs was assessed with flow cytometry, a membrane-based antibody array and enzyme-linked immunosorbant assays. The mineralization capacity of osteoblasts was studied with an assay measuring deposition of hydroxyapatite.

Results: PFD reduced the Ki67 expression 7.1-fold in untreated FLSs $(p=0.001)$ and 11.0-fold in FLSs stimulated with transforming growth factor beta (TGF $\beta)$, tumor necrosis factor alpha (TNFa), and interferon gamma (IFNY) ( $p=0.022)$. There were no statistically significant changes in membrane expression of alpha smooth muscle actin (aSMA), intercellular adhesion molecule 1 (ICAM-1), or human leukocyte antigen DR (HLA-DR). In supernatants from FLSs stimulated with TGF $\beta$, TNFa, and IFNY, PFD decreased the secretion of 3 of 12 proteins more than 2-fold in the membrane-based antibody array. The changes in secretion of monocyte chemoattractant protein 1 (MCP-1) and chitinase-3-like protein $1(\mathrm{CHI} 3 \mathrm{~L} 1, \mathrm{YKL}-40)$ were validated with ELISA. PFD decreased the secretion of both Dickkopf-related protein 1 (DKK1) $(p=0.006)$ and osteoprotegerin (OPG) $(p=0.02)$ by SpA FLSs stimulated with TGF $\beta$, TNFa, and IFN $\gamma$. Finally, PFD inhibited the deposition of hydroxyapatite by osteoblasts in a dose-dependent manner $(p=0.0001)$.

Conclusions: PFD inhibited SpA FLS proliferation and function and osteoblast mineralization in vitro. This encourages studies of the in vivo effect of PFD in SpA.

Keywords: Spondyloarthritis, Inflammation, Fibroblast, Myofibroblast, Osteoblast, Pirfenidone, Fibrosis

\footnotetext{
* Correspondence: kragstrup@biomed.au.dk

${ }^{1}$ Department of Biomedicine, Aarhus University, Aarhus, Denmark

${ }^{4}$ Department of Rheumatology, Aarhus University Hospital, Aarhus, Denmark

Full list of author information is available at the end of the article
}

(c) The Author(s). 2018 Open Access This article is distributed under the terms of the Creative Commons Attribution 4.0 International License (http://creativecommons.org/licenses/by/4.0/), which permits unrestricted use, distribution, and reproduction in any medium, provided you give appropriate credit to the original author(s) and the source, provide a link to the Creative Commons license, and indicate if changes were made. The Creative Commons Public Domain Dedication waiver (http://creativecommons.org/publicdomain/zero/1.0/) applies to the data made available in this article, unless otherwise stated. 


\section{Background}

Spondyloarthritis $(\mathrm{SpA})$ is characterized by inflammation of the axial skeleton and includes ankylosing spondylitis, reactive arthritis, psoriatic arthritis and arthritis associated with inflammatory bowel disease [1,2]. The pathogenesis of SpA involves both inflammation and new bone formation in the spine and the disease has been characterized as both inflammatory and fibrotic $[3,4]$. The inflammatory component causes pain and morning stiffness and can be managed with non-steroidal anti-inflammatory drugs and inhibitors of tumor necrosis factor alpha (TNF $\alpha$ ), interleukin 17 (IL-17) and IL-23. The calcification of the tendons and ligaments and new bone formation leading to ankylosis of the spine causes irreversible compromised range of motion. The retardation of ankylosis has still not been successful $[5,6]$. The role of non-steroidal anti-inflammatory drugs still has to be defined, early and long term treatment seems to be necessary for the TNFa inhibitors and the role of blocking IL-17 and IL-23 is still not clear [7]. Therefore, there is an unmet therapeutic need for the treatment of new bone formation in SpA.

The inflammation in SpA is not fully understood but involves bacterial and mechanical stress $[1,8]$. The most prominent immune abnormality in SpA is the genetic association with human leukocyte antigen B27 (HLA-B27). Further, several proinflammatory membrane molecules with importance for cell adhesion and immune activation are upregulated including intercellular adhesion molecule 1 (ICAM-1). Finally, several cytokines and chemokines are important in the pathogenesis of SpA including macrophage derived TNF $\alpha$, and lymphocyte derived interferon gamma (IFNY) and IL-17 [9], while other molecules have been suggested as biomarkers of diagnosis or treatment response such as chemoattractant protein 1 (MCP-1, CCL2) and chitinase-3-like protein 1 (CHI3L1, YKL-40) $[10,11]$.

Fibrosis is mediated by alpha smooth muscle actin ( $\alpha$ SMA) expressing myofibroblasts as seen in wound healing and fibrotic diseases such as systemic scleroderma and lung fibrosis $[12,13]$. One of the distinct features discriminating spondyloarthritis from rheumatoid arthritis is transforming growth factor beta (TGF $\beta$ ) induced upregulation of myofibroblasts involved in new bone formation at entheseal sites $[4,14]$. Bone metabolism is otherwise the result of increased osteoblast mineralization and decreased osteoclast resorption. A balance between activating bone morphogenetic proteins and inhibiting Dickkopf-1 (DKK1) regulates osteoblast activity. Osteoclasts are activated by the interaction between receptor activator of nuclear factor- $\mathrm{KB}$ (RANK) on osteoclasts and RANK ligand (RANKL), which is blocked by osteoprotegrin (OPG).

Because new bone formation in SpA resemble fibrosis in fibrotic diseases these processes could have similar therapeutic targets and share treatment approaches [3]. Pirfenidone (PFD, brand names Esbriet and Pirespa) is a new drug used to treat idiopathic lung fibrosis. It is an orally active small molecule (MW 185) that is able to move through cell membranes without requiring a receptor. The drug is relatively well tolerated $[15,16]$. PFD has been approved for the treatment of idiopathic lung fibrosis [17]. Further, PFD has shown promising effects in several animal models and in clinical trials of other fibrotic diseases and in a small cohort of patients with rheumatoid arthritis $[15,16,18,19]$.

Here, we evaluate the potential effect of PFD in cell culture models of SpA. We hypothesize that PFD inhibits the formation and activity of spondyloarthritis myofibroblasts and osteoblasts and thereby potentially reduces new bone formation in spondyloarthritis.

\section{Methods}

\section{Study subjects}

A study population consisting of SpA patients $(n=6)$ with peripheral involvement was included for obtaining synovial fluid for growing fibroblast-like synoviocytes (FLSs). Patients with peripheral arthritis contacted the clinic because of a knee joint effusion. No disease activity or prognosis scores or test results were obtained for this study population.

\section{Sample handling}

Synovial fluid mononuclear cells (SFMCs) were isolated by conventional Ficoll-Paque (GE Healthcare) density-gradient centrifugation and cryopreserved at $-135{ }^{\circ} \mathrm{C}$ until time of use.

\section{Fibroblast-like synoviocyte cultures}

FLSs were grown from SFMCs as described previously $[20,21]$. Briefly, SFMCs were thawed and cultured in Dulbecco's modified Eagle medium (Lonza) supplemented with $10 \%$ fetal bovine serum (FBS), penicillin, streptomycin, and glutamine at $37{ }^{\circ} \mathrm{C}$ and $5 \% \mathrm{CO}_{2}$ at a density of $2 \times 10^{6}$ cells $/ \mathrm{ml}$ in a humidified incubator, replacing the medium every 3 to 4 days. When the cell layer was $70 \%$ confluent, the FLSs were passaged by trypsin treatment and used for analyses at passage 4-5. FLSs were stimulated with either TGF $\beta$ at $5 \mathrm{ng} / \mathrm{ml}, \mathrm{TNF} \alpha$ at $10 \mathrm{ng} / \mathrm{ml}$, and IFNY at $10 \mathrm{ng} / \mathrm{ml}$ alone or in combination and cultured with or without PFD at $1.0 \mathrm{mg} / \mathrm{ml}$ for $48 \mathrm{~h}$. This concentration has previously been shown not to induce apoptosis or cell death in fibroblasts from several anatomical sights including FLSs [22-25]. Supernatants were harvested and kept at $-80^{\circ} \mathrm{C}$ until time of use.

\section{Flow cytometry}

SpA FLSs were cultured at a density of $5.0 \times 10^{4}$ cells $/ \mathrm{ml}$ in RPMI-1640 (Lonza) supplemented with 10\% FBS, 
penicillin, streptomycin, and glutamine at $37{ }^{\circ} \mathrm{C}$ and $5 \%$ $\mathrm{CO}_{2}$ in a humidified incubator for $24 \mathrm{~h}$. Cells were stimulated with either TGF $\beta$ at $5 \mathrm{ng} / \mathrm{ml}$, TNF $\alpha$ at $10 \mathrm{ng} / \mathrm{ml}$, and IFNY at $10 \mathrm{ng} / \mathrm{ml}$ alone or in combination and cultured with or without PFD at $1.0 \mathrm{mg} / \mathrm{ml}$ for $48 \mathrm{~h}$. Cells were then harvested by trypsin/EDTA treatment, transferred to polypropylene tubes (Nunc) and fixed using $4 \%$ formaldehyde (Sigma Aldrich) diluted in PBS. Cells were then permeabilized using 0.3\% saponin (Sigma Aldrich) in PBS with $0.5 \%$ bovine serum albumin (BSA) (Calbiochem) and $0.09 \% \mathrm{NaN}_{3}$. Staining with antibodies was done in a buffer containing $10 \mu \mathrm{g} / \mathrm{ml}$ mouse gamma globulin (Jackson ImmunoResearch) to minimize non-specific binding [26]. Cell surface staining before permeabilization was done with anti-CD90 phycoerythrin cyanine 7 (PC7) (BioLegend), anti-ICAM-1 allophycocyanin (APC) (BD), and anti-HLA-DR phycoerythrin (PE) (BD) with incubation 30 min at $4{ }^{\circ} \mathrm{C}$. Intracellular staining after permeabilization was done with anti-Ki67 alexa488 (BioLegend) and anti- $\alpha$ SMA APC (R\&D Systems) with incubation $30 \mathrm{~min}$ at $4{ }^{\circ} \mathrm{C}$. Dead cells were excluded based on staining with Live/Dead fixable viability marker (near-infra red, Life Technologies). The samples were analysed using an LSR Fortessa flow cytometer (BD Biosciences) and data analysed using FlowJo software version 10 (Tree Star Inc.).

\section{Membrane-based antibody array}

Culture supernatants were analysed with a membranebased antibody array for the parallel determination of the relative levels of cytokines and chemokines as done previously (Proteome Profiler ${ }^{\mathrm{TM}}$ Human XL Cytokine Array Kit, R\&D Systems) [27].

\section{Enzyme linked immunosorbant assay}

Culture supernatants were analysed with a commercially available MCP-1 (Biolegend), YKL-40 (R\&D Systems), RANKL (R\&D Systems), DKK-1 (R\&D Systems), OPG (R\&D Systems) enzyme-linked immunosorbant assays (ELISAs) following manufacturer's instructions.

\section{Osteoblast cultures}

The osteoblast cell line Saos-2 was used. The cells were cultured and expanded in supplemented osteoblast growth medium (C-27001, PromoCell). The cells were seeded in triplicates in 96-well plates at a concentration of 100,000 cells/ml (20,000 cells/well). Cells were then cultured in osteoblast mineralization medium (C-27020, PromoCell) with PFD either at $0.25 \mathrm{mg} / \mathrm{ml}, 0.5 \mathrm{mg} / \mathrm{ml}$, or $1.0 \mathrm{mg} / \mathrm{ml}$ for 14 days. A negative control culture without PFD was used in each experiment for comparison. Medium with mediators was changed every 2-3 days. On day 14 the formed mineral was visualized using a commercial mineralization stain kit (OsteoImage, PA-1503, Lonza), which conjugates a fluorophore to hydroxyapatite in the mineral. Finally, the degree of mineralization was quantified using a plate reader (Thermo Scientific, Fluoroscan Ascent FL) $[28,29]$.

\section{Statistics}

All flow cytometry, ELISA and mineralization measurements and clinical scores were expressed with the median and interquartile range (IQR). Flow cytometry, ELISA and mineralization measurements ratios were log-transformed and comparisons were made with the paired t-test or the repeated measures one-way ANOVA depending on the number of groups. A two-tailed $P$-value below 0.05 was considered statistically significant. Calculations and graphs were made with Stata (StataCorp LP) and GraphPad Prism (GraphPad Software).

\section{Results}

\section{Proliferation of SpA FLSs}

We first evaluated the effect of PFD on fibroblast proliferation. PFD decreased the number of fibroblasts after $24 \mathrm{~h}$ and $72 \mathrm{~h}$ of culture without causing detachment of cells as evaluated by light microscopy (Fig. 1a and b). This was seen in both untreated cells and cells stimulated with TGF $\beta$, TNF $\alpha$, and IFN $\gamma$. The median percentage of dead cells measured with the Live/Dead fixable viability marker by flow cytometry was $0.31 \%$ (IQR $0.010 \%$ to $0.57 \%$ ) for untreated cells and $0.20 \%$ (IQR $0.035 \%$ to $0.28 \%$ ) for pirfenidone treated cells (Fig. 1c). The decreased proliferation was quantified by measuring Ki67 expression with flow cytometry (Fig. 1d and e). PFD reduced the Ki67 expression 7.1-fold in untreated cells $(p=0.001)$ and 11.0-fold in cells stimulated with TGF $\beta$, TNF $\alpha$, and IFN $\gamma(p=0.022)$ (Fig. 1f).

\section{Expression of intracellular aSMA and membrane HLA-DR and ICAM-1 in SpA FLSs}

We now studied whether PFD could alter expression of intracellular and membrane molecules characterizing FLS differentiation and activity. We studied the induction of three molecules known to be induced by IFN $\gamma$, TNF $\alpha$, and TGF $\beta$. These were the major histocompatibility complex HLA-DR, ICAM-1, and $\alpha$ SMA, respectively. The TGF $\beta$ induced upregulation of $\alpha$ SMA (Fig. 2a and $b$ ) and the IFN $\gamma$ induced upregulation of HLA-DR (Fig. 2c and d) were not significantly decreased by PFD treatment. There was no difference between the percentage of ICAM-1 positive FLSs in TNF $\alpha$ stimulated cultures treated with or without PFD (Fig. 2e and f).

\section{Secretion of cytokines and chemokines from SpA FLSs}

We then examined the effect of PFD on the secretion of cytokines and chemokines by SpA FLSs. We used a membrane-based antibody array for the determination of a large panel of cytokines and chemokines secreted by 


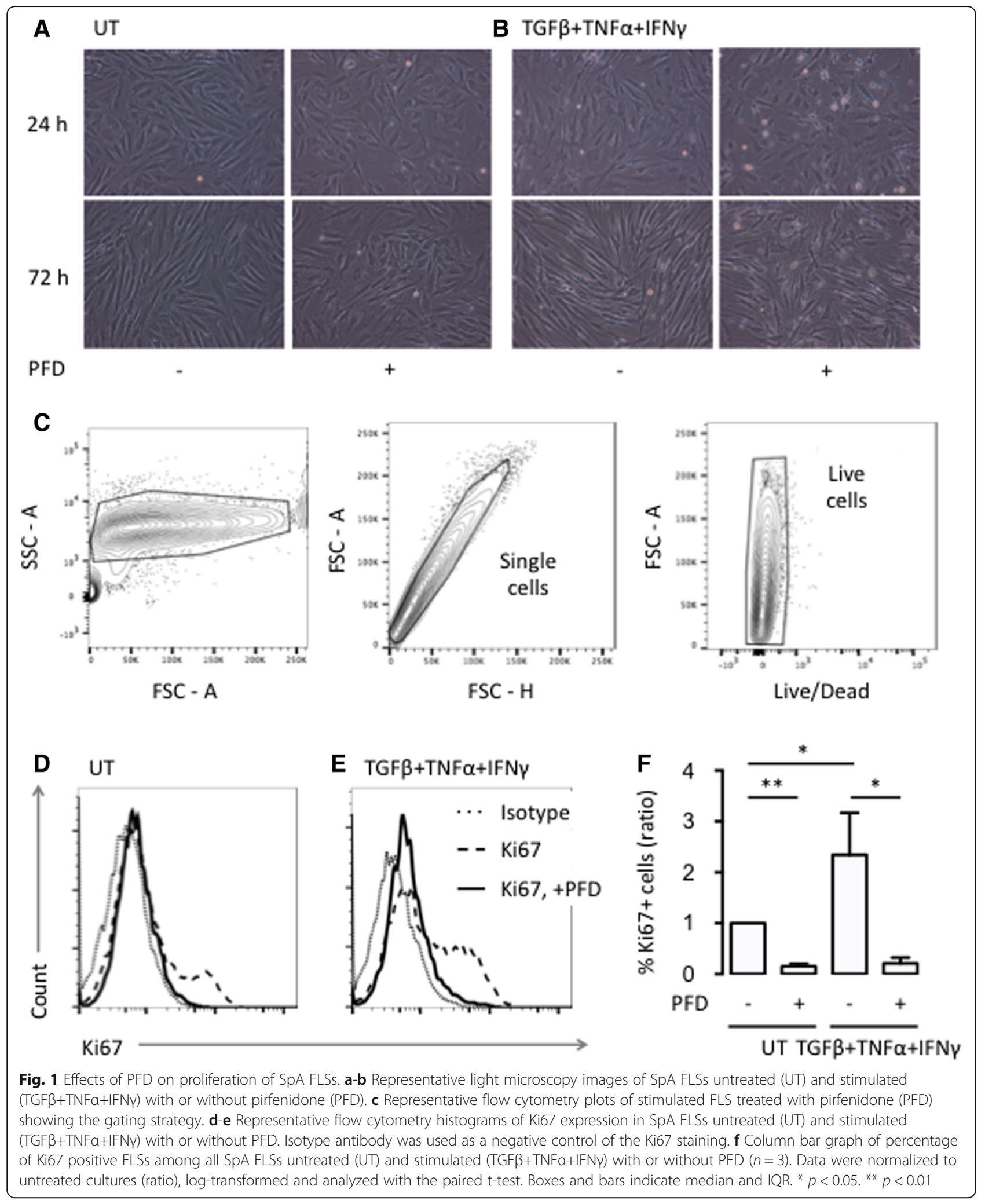

SpA FLSs stimulated with both TGF $\beta$, TNF $\alpha$, and IFN $\gamma$ with or without PFD. A total of 12 cytokines or chemokines had values above the detection limit measured as staining on the membrane array relative to the reference spots. PFD decreased the secretion of insulin-like growth factor-binding protein 3 (IGFBP-3), monocyte-specific 


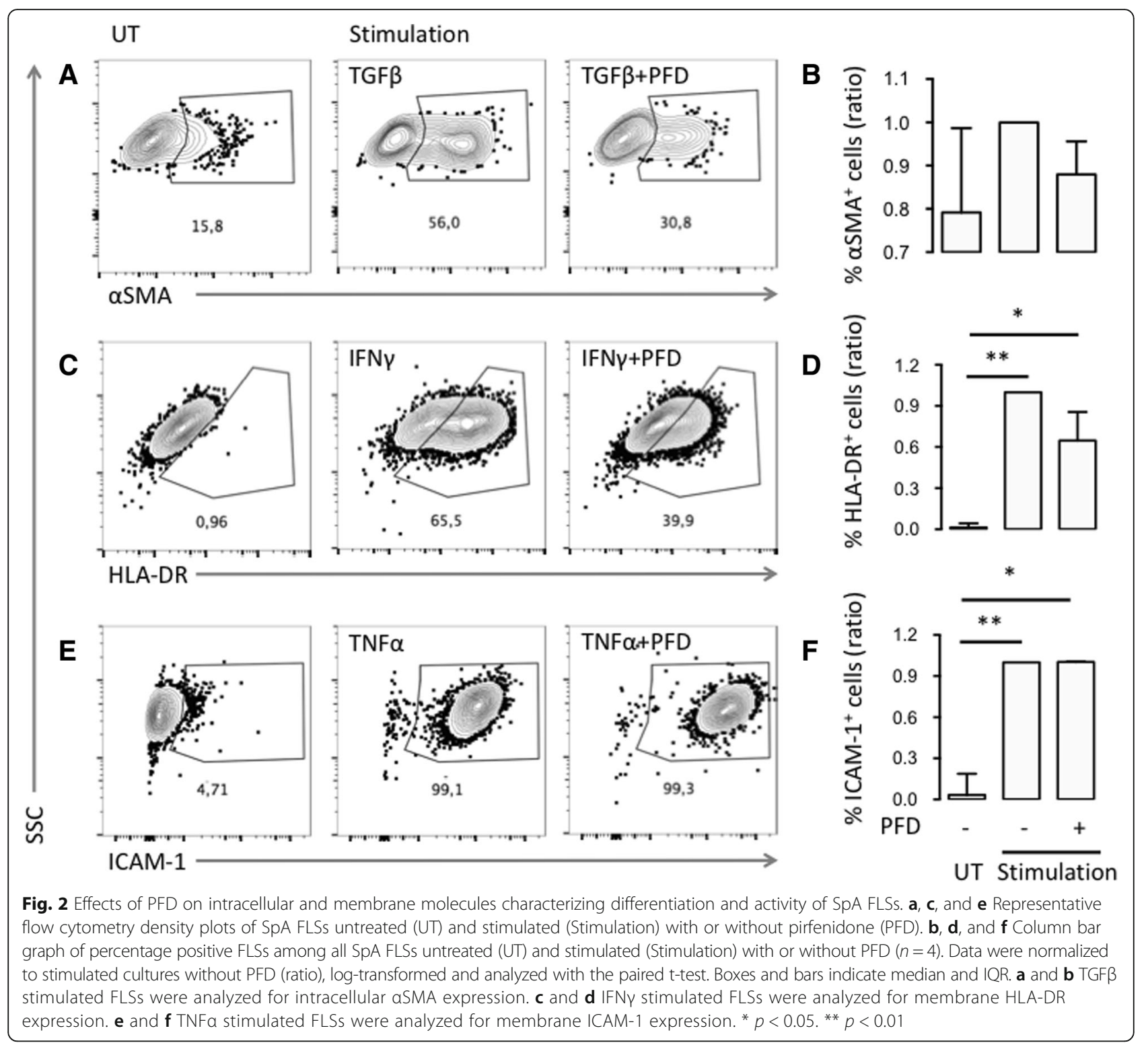

chemokine 3 (MCP-3, also called chemokine (C-C motif) ligand 7 (CCL7)), and YKL-40 (also called chitinase-3-like protein 1 (CHI3L1)) more than 2-fold (Fig. 3a and b and Table 1). The changes in secretion of MCP-1 and YKL-40 were validated with ELISA showing similar results. Thus, PFD resulted in no or a modest decrease in MCP-1 secretion and a significant decrease in YKL-40 secretion in SpA FLSs stimulated with both TGF $\beta$, TNF $\alpha$, and IFN $\gamma$ (Fig. 3c and d). However, PFD decreased both MCP-1 and YKL-40 in SpA FLSs cultured without stimulation or stimulated with TNF $\alpha$ or IFN $\gamma$ alone (Fig. $3 \mathrm{c}$ and d).

\section{Secretion of bone homeostasis cytokines from SpA FLSs} and osteoblast mineralization

We finally tested whether PFD could interfere with secretion of regulators of bone metabolism by SpA FLSs and osteoblast mineralization. Secretion of the osteoclast activator RANKL, the osteoclast inhibitor OPG, and the osteoblast inhibitor DKK-1 by SpA FLSs was analyzed. PFD decreased the secretion of both DKK1 $(p=0.006)$ and OPG $(p=0.02)$ by SpA FLSs stimulated with a combination of TGF $\beta$, TNF $\alpha$, and IFN $\gamma$ (Fig. 4a and b). The concentration of RANKL was below the detection limit of the ELISA assay in all cultures. The mineralization assay was done with Saos-2 cells incubated with increasing concentrations of PFD for 14 days. PFD inhibited the deposition of hydroxyapatite by Saos- 2 cells in a dose-dependent manner $(p=0.0001)$ (Fig. 4c).

\section{Discussion}

The pathogenesis of SpA involves both inflammation and new bone formation in the spine. The treatment 


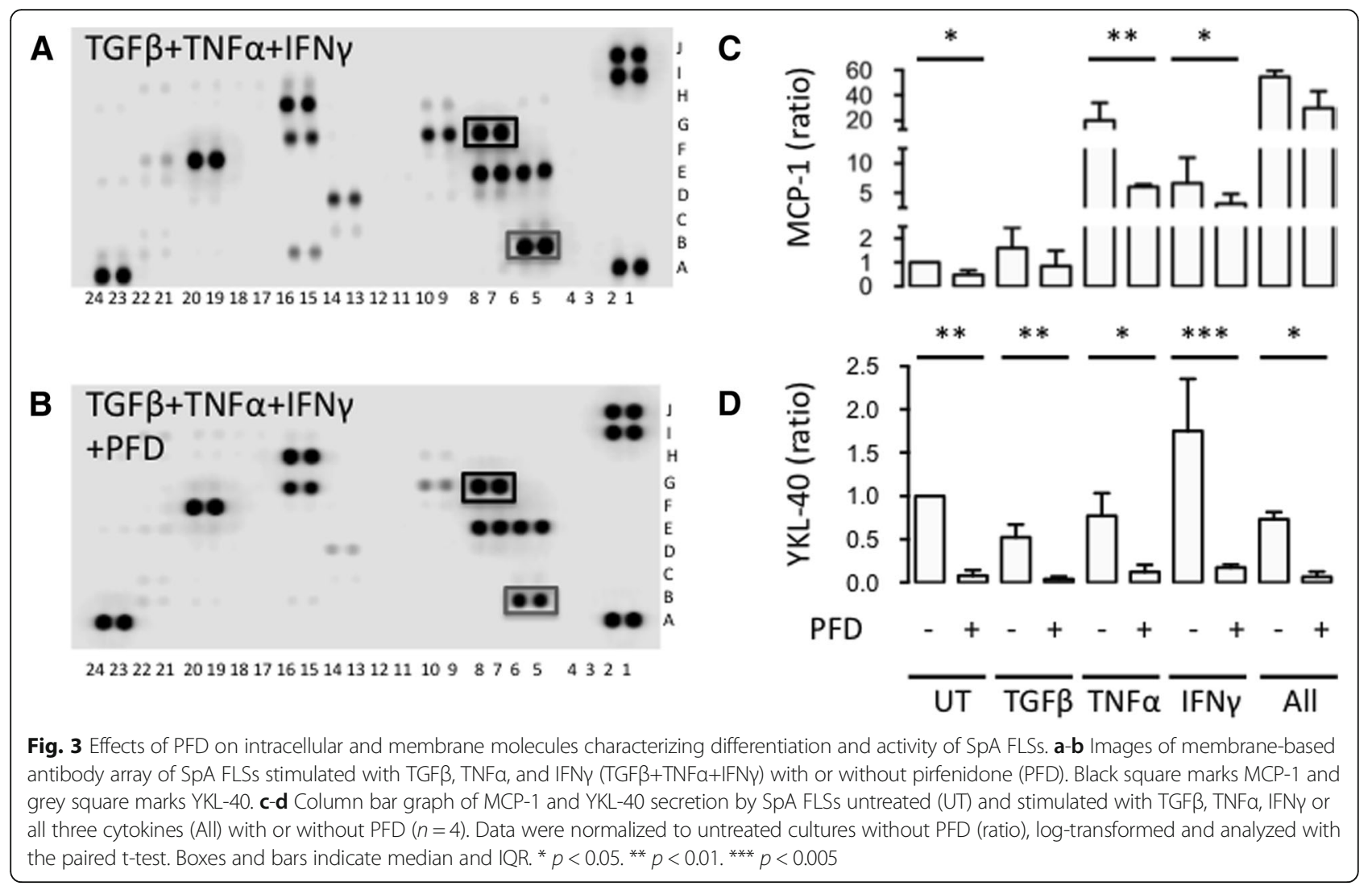

Table 1 Effect of PFD on the secretion of cytokines and chemokines from SpA FLSs

\begin{tabular}{ll}
\hline Cytokine/chemokine & Fold decrease \\
\hline IGFBP-3 & $\mathbf{3 . 6 4}$ \\
MCP-3 & $\mathbf{2 . 8 8}$ \\
YKL-40 & $\mathbf{2 . 3 2}$ \\
IL-8 & 1.30 \\
MIG & 1.27 \\
MCP-1 & 1.23 \\
Serpin E1 & 1.21 \\
IL-6 & 1.13 \\
IP-10 & 1.08 \\
RANTES & 1.04 \\
Trombospondin-1 & 1.02 \\
ICAM-1 & 1.02
\end{tabular}

Using Image StudioTM 4.0 (LI-COR Biosciences UK Ltd) the average pixel density of the duplicate spots was determined along with the three pairs of reference spots on each array. The fold decrease is the ratio of the value of untreated cells divided by the value of pirfenidone treated cells $(n=1)$. IGFBP-3; Insulin-like growth factor-binding protein 3. MCP-3; monocyte-specific chemokine 3 (also called chemokine (C-C motif) ligand 7 (CCL7)). YKL-40 (also called chitinase-3-like protein 1 (CHI3L1)). MIG; monokine induced by gamma interferon (also called chemokine (C-X-C motif) ligand 9 (CXCL9)). Serpin E1 (also called plasminogen activator inhibitor-1 (PAI-1)). IP-10; interferon gamma-induced protein 10 (also called C-X-C motif chemokine 10 (CXCL10)). RANTES; regulated on activation, normal T cell expressed and secreted (also called chemokine (C-C motif) ligand 5 (CCL5)). ICAM-1; Intercellular Adhesion Molecule 1. Bold text and numbers indicate fold change $>2$. Italic text and numbers indicate validation with ELISA with non-steroidal anti-inflammatory drugs and inhibitors of TNF $\alpha$, IL-17 and IL-23 dampens inflammation while new bone formation can progress. Therefore, there is an unmet therapeutic need for the treatment of new bone formation in SpA. Here, we found that PFD inhibits SpA fibroblasts proliferation and cytokine secretion and osteoblast mineralization. Therefore, PFD could be a novel inhibitor of new bone formation in SpA.

In $\mathrm{SpA}$, the purpose of the treatment is to induce clinical remission and prevent radiographic progression. Now, all treatment strategies for SpA are based on suppressing or modulating the immune system, and the agents currently used may induce clinical remission but do not satisfyingly prevent radiographic progression. The radiographic progression is caused by fibrosis and new bone formation. The pathogenesis of fibrosis is poorly understood. TGF $\beta$ is the prototypical profibrotic cytokine involved in fibrosis in many organ systems. TGF $\beta$ stimulated cells undergo a phenotypical transformation becoming activated myofibroblasts that express contractile proteins such as $\alpha$ SMA. Therefore, the marked upregulation of myofibroblasts in SpA and link to new bone formation is interesting [4, 14, 30, 31]. New bone formation is the result of increased osteoblast mineralization and decreased osteoclast resorption. 


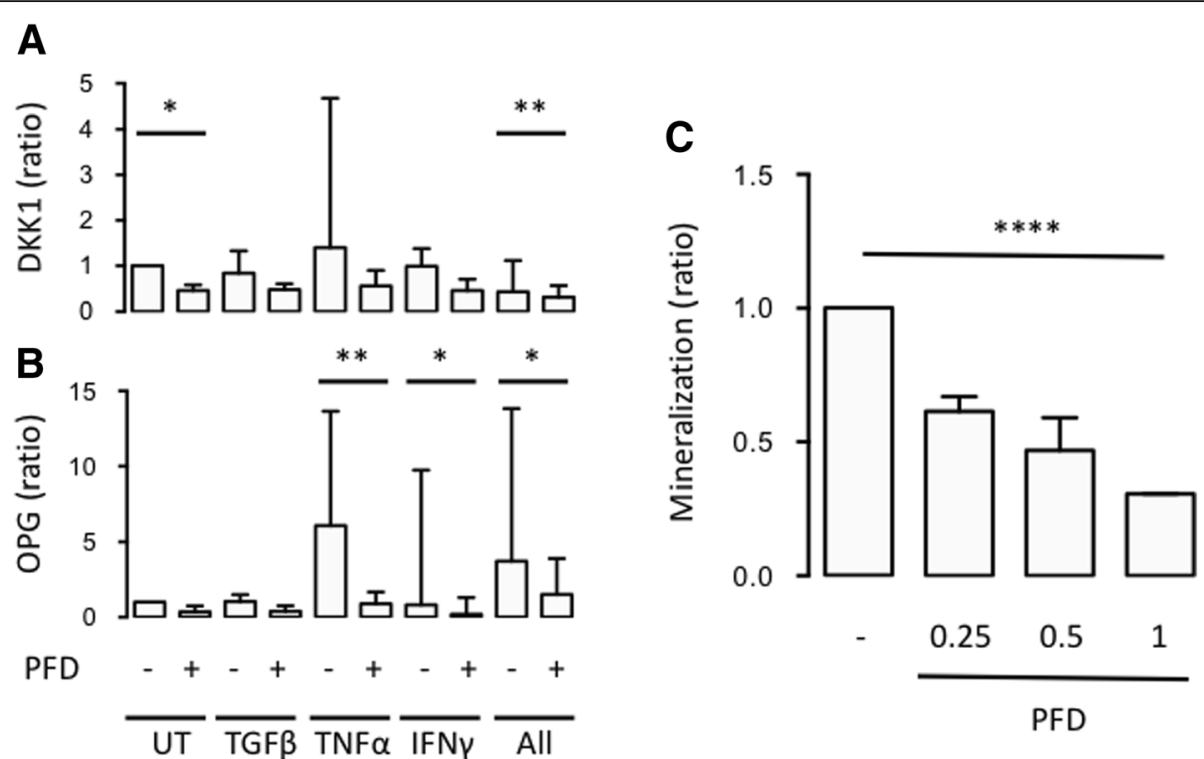

Fig. 4 Effects of PFD on secretion of regulators of bone metabolism by SpA FLSs and osteoblast mineralization. a-b Column bar graph of DKK-1 and OPG secretion by SpA FLSs untreated (UT) and stimulated with TGF $\beta$, TNFa, IFNY or all three cytokines (All) with or without pirfenidone (PFD) $(n=4)$. Data were normalized to untreated cultures without PFD (ratio), log-transformed and analyzed with the paired t-test. c Column bar graph of hydroxyapatite deposition by Saos-2 cells incubated with PFD (triplicates). Saos-2 cells were incubated with increasing concentrations of PFD for 14 days. Data were normalized to untreated cultures without PFD (ratio), log-transformed and comparisons were made with the paired t-test or the repeated measures one-way ANOVA depending on the number of groups. Boxes and bars indicate median and IQR. ${ }^{*} p<0.05 .{ }^{* *} p<0.01 .{ }^{* *} p<0.005$

PFD exhibits well-documented antifibrotic, anti-inflammatory, and antioxidant activities in a variety of animal and cell-based models, although its molecular target has not been elucidated. In vitro, PFD inhibits the proliferation and activation of a broad variety of cells including fibroblasts, leiomyoma cells, and T cells $[15,16]$. In animal models PFD reduces fibrosis in the lung, liver, heart, and kidney [15, 18]. In 2011, PFD was approved for the treatment of idiopathic pulmonary fibrosis [17]. The drug has also been tested in studies with patients with rheumatoid arthritis without serious side effects [19].

In this study, PFD showed both anti-inflammatory and possible anti-fibrotic effects on SpA FLSs. First, the effect of PFD on FLS proliferation and expression of the myofibroblast marker $\alpha$ SMA was studied. PFD inhibited the proliferation of SpA FLSs. Our finding of decreased proliferation in SpA FLSs is in line with previous studies showing inhibition of proliferation of fibroblasts from lung, striated muscle, heart and eye [22, 23, 32, 33]. The effect was seen in both unstimulated cells and in cells under the influence of TGF $\beta$, TNF $\alpha$, and IFN $\gamma$ simulating an inflammatory environment. The antiproliferative effect of PFD is not completely understood. PFD has been shown to induce apoptosis in hepatocellular carcinoma cells [34]. In vivo, PFD was found to ameliorate ciclosporine nephrotoxicity by decreasing pro-apoptotic genes and to prevent TNF $\alpha$ induced liver injury $[35,36]$. Any effect of PFD on apoptosis of SpA FLS cannot be evaluated from this study. In this study there was no statistically significant suppression of $\alpha$ SMA. However, PFD seemed to have a mild effect to suppress $\alpha$ SMA. This could be interesting because myofibroblast differentiation and formation of extracellular matrix at the entheses seems to be important in SpA [3]. PFD has previously been shown to decrease myofibroblast differentiation and extracellular matrix deposition in fibroblasts from the eye and lung by interfering with the TGF $\beta$ signaling pathway $[37,38]$. Whether PFD alters myofibroblast formation or extracellular matrix secretion cannot be concluded by this study.

Second, the effect of PFD on SpA FLS expression of membrane molecules and secretion of cytokines and chemokines was studied. Previously, PFD has been shown to inhibit MHC-II molecules in animal models of transplantation [39]. In this study, there was no statistically significant suppression of HLA-DR. However, PFD seemed to have a mild effect to suppress IFN $\gamma$ induced expression of HLA-DR on SpA FLSs. Therefore, no final conclusions on the effect of PFD on MHC-II expression by SpA FLSs can be made. PFD has also previously been shown to inhibit IL-1 $\beta$ induced expression of ICAM-1 [25]. However, in this study TNFa induced expression of ICAM-1 was not changed in any way by PFD. The discrepancy could be the lower concentrations of PFD used in this study. PFD has also previously been found to decrease the secretion of several cytokines and chemokines such as IL-6, IL-1 $\beta$, and MCP-1 in animal models of fibrotic disease [18]. This study confirms some of these 
findings and adds novel targets to the list of inflammatory mediators decreased by PFD. Especially the finding of PFD decreased YKL-40 is interesting because this mediator has been associated with both fibrotic diseases such as pulmonary fibrosis and liver fibrosis and spondyloarthritis $[11,40]$. There were large inter-donor variations. Therefore, it is not possible to conclude whether PFD is more effective in preventing MCP-1 or YKL-40 secretion under influence of TGF $\beta$, TNF $\alpha$, IFN $\gamma$ or a combination of all three cytokines.

Third, the effect of PFD on osteoblast mineralization and FLS secretion of bone homeostasis proteins was studied. PFD reduced mineralization by osteoblasts. This study is to our knowledge the first to associate PFD with decreased osteoblast activity. In contrast, PFD also tended to decrease DKK-1, which might result in increased osteoblastogenesis. The surmised effect of PFD on osteoblasts in vivo is therefore to be clarified. FLS secretion of OPG, an inhibitor of osteoclastogenesis, was also reduced by PFD. PFD could therefore increase the number of osteoclasts and thus bone resorption. Further studies are needed to validate the effect of PFD on osteoblasts and osteoclasts.

\section{Conclusion}

PFD inhibited SpA FLS proliferation and cytokine production and osteoblast mineralization in vitro. This study encourages studies of the in vivo effect of PFD in SpA.

\section{Abbreviations \\ CCL2: Chemokine (C-C motif) ligand 2; CHI3L1: Chitinase-3-like protein 1; DKK1: Dickkopf-related protein 1; FBS: Fetal bovine serum; FLS: Fibroblast-like synovial cell; HC: Healthy control; HLA: Human leukocyte antigen; ICAM: Intercellular adhesion molecule; IFN: Interferon; IL: Interleukin; IQR: Interquartile range; LPS: Lipopolysaccharide; MCP-1: Monocyte chemoattractant protein 1; OPG: Osteoprotegerin; PBS: Phosphate buffered saline; PFD: Pirfenidone; RANK: Receptor activator of nuclear factor-KB; SFMC: Synovial fluid mononuclear cell; SMA: Smooth muscle actin; SpA: Spondyloarthrtis; TGF: Transforming growth factor; TNF: Tumour necrosis factor; UT: Untreated; YKL40 (CHI3L1): Chitinase-3-like protein 1}

\section{Acknowledgements}

We thank Karin Skovgaard Sørensen at the Department of Biomedicine, Aarhus University for excellent technical assistance concerning the ELISA systems and the mineralization assay. We thank medical doctors and nurses at the Department of Rheumatology, Aarhus University Hospital for helping collect the patient samples. All flow cytometric analyses were performed using the LSR Fortessa at the FACS core facility at Aarhus University.

\section{Funding}

The Danish Rheumatism Association, Aage Bangs Foundation, the Nyegaard Foundation, the Augustinus Foundation, the Bjarne Jensen Foundation, and the Faculty of Health at Aarhus University funded this work.

\section{Availability of data and materials}

The datasets used and/or analysed during the current study are available from the corresponding author on reasonable request.

\section{Authors' contributions}

TWK helped to design the study, helped to collect the SFMC samples, helped to carry out the experiments, analysed and interpreted the data and drafted the manuscript. JS finalized the manuscript and helped to plan and carry out the experiments. SL and PO helped to plan and carry out the experiments. JK helped to design the study. All authors helped to analyse and interpret the data, were involved in revising the manuscript, and read and approved the final manuscript.

\section{Ethics approval and consent to participate}

Samples were collected at the outpatient clinic at Aarhus University Hospital. All samples were obtained after informed written consent according to the Declaration of Helsinki and approved by the Local Ethics Committee (project number 20121329) and the Danish Data Protection Agency.

\section{Consent for publication}

Not applicable.

\section{Competing interests}

TWK is an Editorial Board Member for BMC Rheumatology. The authors' declare that they have no competing interests.

\section{Publisher's Note}

Springer Nature remains neutral with regard to jurisdictional claims in published maps and institutional affiliations.

\section{Author details}

${ }^{1}$ Department of Biomedicine, Aarhus University, Aarhus, Denmark. ${ }^{2}$ Department of Dermatology, Aarhus University Hospital, Aarhus, Denmark. ${ }^{3}$ Department of Hepatology and Gastroenterology, Aarhus University Hospital, Aarhus, Denmark. ${ }^{4}$ Department of Rheumatology, Aarhus University Hospital, Aarhus, Denmark. ${ }^{5}$ Department of Internal Medicine, Randers Regional Hospital, Randers, Denmark.

Received: 22 May 2018 Accepted: 14 October 2018

Published online: 19 November 2018

\section{References}

1. Dougados M, Baeten D. Spondyloarthritis. Lancet. 2011;377:2127-37.

2. Taurog JD, Chhabra A, Colbert RA. Ankylosing spondylitis and axial Spondyloarthritis. N Engl J Med. 2016;374:2563-74.

3. Beyer C, Distler JHW. Changing paradigms in spondylarthritis: the myofibroblast signature. Arthritis Rheum. 2012;65:24-7.

4. Jacques P, Lambrecht S, Verheugen E, Pauwels E, Kollias G, Armaka M, et al. Proof of concept: enthesitis and new bone formation in spondyloarthritis are driven by mechanical strain and stromal cells. Ann Rheum Dis. 2013;73: 437-45

5. Baraliakos $X$, Haibel $H$, Listing J, Sieper J, Braun J. Continuous long-term anti-TNF therapy does not lead to an increase in the rate of new bone formation over 8 years in patients with ankylosing spondylitis. Ann Rheum Dis. 2014:73:710-5.

6. Lories RJU, Luyten FP, de Vlam K. Progress in spondylarthritis. Mechanisms of new bone formation in spondyloarthritis. Arthritis Res Ther. 2009:11:221

7. Maksymowych WP, Morency N, Conner-Spady B, Lambert RG. Suppression of inflammation and effects on new bone formation in ankylosing spondylitis: evidence for a window of opportunity in disease modification. Ann Rheum Dis. 2013;72:23-8.

8. Ambarus C, Yeremenko N, Tak PP, Baeten D. Pathogenesis of spondyloarthritis. Curr Opin Rheumatol. 2012;24:351-8.

9. Hreggvidsdottir HS, Noordenbos T, Baeten DL. Inflammatory pathways in spondyloarthritis. Mol Immunol. 2014;57:28-37.

10. Romero-Sanchez C, Tsou H-K, Jan M-S, Wong R-H, Chang I-C, Londoño J, et al. Serum monocyte chemotactic protein-1 concentrations distinguish patients with ankylosing spondylitis from patients with mechanical low back pain. J Spinal Disord Tech. 2011;24:202-7.

11. Pedersen SJ, Hetland ML, Sørensen IJ, Ostergaard M, Nielsen HJ, Johansen JS. Circulating levels of interleukin-6, vascular endothelial growth factor, YKL-40, matrix metalloproteinase-3, and total aggrecan in spondyloarthritis patients during 3 years of treatment with TNFa inhibitors. Clin Rheumatol. 2010;29:1301-9.

12. Gabrielli A, Avvedimento EV, Krieg T. Scleroderma. N Engl J Med. 2009;360: 1989-2003.

13. Li J, Chen J, Kirsner R. Pathophysiology of acute wound healing. Clin Dermatol. 2007;25:9-18. 
14. Yeremenko N, Noordenbos T, Cantaert T, van Tok M, van de Sande M, Cañete JD, et al. Disease-specific and inflammation-independent stromal alterations in spondyloarthritis synovitis. Arthritis Rheum. 2012;65:174-85.

15. Macías-Barragán J. The multifaceted role of pirfenidone and its novel targets. Fibrogenesis Tissue Repair. 2010;3:16.

16. Lopez-de la Mora DA, Sanchez-Roque C, Montoya-Buelna M, SanchezEnriquez S, Lucano-Landeros S, Macias-Barragan J, et al. Role and new insights of Pirfenidone in fibrotic diseases. Int J Med Sci. 2015;12:840-7.

17. Cottin $\mathrm{V}$. The role of pirfenidone in the treatment of idiopathic pulmonary fibrosis. Respir Res. 2013;14(Suppl 1):S5.

18. Schaefer CJ, Ruhrmund DW, Pan L, Seiwert SD, Kossen K. Antifibrotic activities of pirfenidone in animal models. Eur Respir Rev. 2011;20:85-97.

19. $E P E S, S N G, S B M$. Antirheumatic effect of pirfenidone in a double blind clinical pilot trial in humans. Res Commun Mol Pathol Pharmacol. 2004;115116:39-48.

20. Kragstrup TW, Jalilian B, Hvid M, Kjærgaard A, Østgård R, Schiøttz Christensen B, et al. Decreased plasma levels of soluble CD18 link leukocyte infiltration with disease activity in spondyloarthritis. Arthritis Res. Ther. 2014;16:R42.

21. Stebulis JA, Rossetti RG, Atez FJ, Zurier RB. Fibroblast-like synovial cells derived from synovial fluid. J Rheumatol. 2005;32:301-6.

22. Shi Q, Liu X, Bai Y, Cui C, Li J, Li Y, et al. In vitro effects of pirfenidone on cardiac fibroblasts: proliferation, myofibroblast differentiation, migration and cytokine secretion. PLoS One. 2011;6:e28134.

23. Zanotti S, Bragato C, Zucchella A, Maggi L, Mantegazza R, Morandi L, et al. Anti-fibrotic effect of pirfenidone in muscle derived-fibroblasts from Duchenne muscular dystrophy patients. Life Sci. 2016;145:127-36.

24. Kadir SI, Wenzel Kragstrup T, Dige A, Kok Jensen S, Dahlerup JF, Kelsen J. Pirfenidone inhibits the proliferation of fibroblasts from patients with active Crohn?S disease. Scand J Gastroenterol. 2016;51:1321-5.

25. Kaneko M, Inoue H, Nakazawa R, Azuma N, Suzuki M, Yamauchi S, et al. Pirfenidone induces intercellular adhesion molecule-1 (ICAM-1) downregulation on cultured human synovial fibroblasts. Clin Exp Immunol. 1998; 113:72-6.

26. Andersen MN, Al-Karradi SNH, Kragstrup TW, Hokland M. Elimination of erroneous results in flow cytometry caused by antibody binding to fc receptors on human monocytes and macrophages. Cytometry Part A. 2016; 89:1001-9.

27. Ommen P, Stjernholm T, Kragstrup T, Raaby L, Johansen C, Stenderup K, et al. The role of leptin in psoriasis comprises a proinflammatory response by the dermal fibroblast. Br J Dermatol. 2016:174:187-90.

28. Heftdal LD, Andersen T, Jæhger D, Woetmann A, Østgård R, Kenngott EE, et al. Synovial cell production of IL-26 induces bone mineralization in spondyloarthritis. J Mol Med. 2017;95:779-87.

29. Kragstrup TW, Andersen MN, Schiøttz-Christensen B, Jurik AG, Hvid M, Deleuran B. Increased interleukin (IL)-20 and IL-24 target osteoblasts and synovial monocytes in spondyloarthritis. Clin Exp Immunol. 2017;189:342-51.

30. Sieper J, Appel H, Braun J, Rudwaleit M. Critical appraisal of assessment of structural damage in ankylosing spondylitis: implications for treatment outcomes. Arthritis Rheum. 2008:58:649-56.

31. Maksymowych WP, Chiowchanwisawakit P, Clare T, Pedersen SJ, Ostergaard M, Lambert RGW. Inflammatory lesions of the spine on magnetic resonance imaging predict the development of new syndesmophytes in ankylosing spondylitis: evidence of a relationship between inflammation and new bone formation. Arthritis Rheum. 2009;60:93-102.

32. Lee $\mathrm{K}$, Young Lee $\mathrm{S}$, Park SY, Yang H. Antifibrotic effect of pirfenidone on human pterygium fibroblasts. Curr Eye Res. 2014;39:680-5.

33. Conte $\mathrm{E}$, Gili $\mathrm{E}$, Fagone $\mathrm{E}$, Fruciano M, lemmolo M, Vancheri C. Effect of pirfenidone on proliferation, TGF- $\beta$-induced myofibroblast differentiation and fibrogenic activity of primary human lung fibroblasts. Eur J Pharm Sci. 2014;58:13-9.

34. Zou W-J, Huang Z, Jiang T-P, Shen Y-P, Zhao A-S, Zhou S, et al. Pirfenidone inhibits proliferation and promotes apoptosis of hepatocellular carcinoma cells by inhibiting the Wnt/ $\beta$-catenin signaling pathway. Med Sci Monit. 2017;23:6107-13

35. Komiya C, Tanaka M, Tsuchiya K, Shimazu N, Mori K, Furuke S, et al. Antifibrotic effect of pirfenidone in a mouse model of human nonalcoholic steatohepatitis. Sci Rep. 2017;7:44754.

36. Shihab FS, Bennett WM, Yi H, Andoh TF. Effect of pirfenidone on apoptosisregulatory genes in chronic cyclosporine nephrotoxicity. Transplantation. 2005;79:419-26.
37. Kurita Y, Araya J, Minagawa S, Hara H, Ichikawa A, Saito N, et al. Pirfenidone inhibits myofibroblast differentiation and lung fibrosis development during insufficient mitophagy. Respir Res. 2017;18:114.

38. Stahnke T, Kowtharapu BS, Stachs O, Schmitz K-P, Wurm J, Wree A, et al. Suppression of TGF- $\beta$ pathway by pirfenidone decreases extracellular matrix deposition in ocular fibroblasts in vitro. PLoS One. 2017;12:e0172592.

39. Bizargity P, Liu K, Wang L, Hancock WW, Visner GA. Inhibitory effects of pirfenidone on dendritic cells and lung allograft rejection. Transplantation. 2012:94:114-22.

40. Lee CG, Da Silva CA, Cruz Dela CS, Ahangari F, Ma B, Kang M-J, et al. Role of chitin and chitinase/chitinase-like proteins in inflammation, tissue remodeling, and injury. Annu Rev Physiol. 2011;73:479-501.
Ready to submit your research? Choose BMC and benefit from:

- fast, convenient online submission

- thorough peer review by experienced researchers in your field

- rapid publication on acceptance

- support for research data, including large and complex data types

- gold Open Access which fosters wider collaboration and increased citations

- maximum visibility for your research: over $100 \mathrm{M}$ website views per year

At BMC, research is always in progress.

Learn more biomedcentral.com/submissions 